\title{
METEOROLOGICAL AND CHEMICAL PREDICTION WEB BASED SYSTEM USING RAMS AND CAMX NUMERICAL MODELS
}

\author{
M. Victoria. Toro, \\ Nestor W. Alvarez Villa and \\ Carlos G. Sanchez Toro \\ Environmental Research Group (GIA) \\ Bolivariana Pontifical University (UPB) \\ Circ. 1a 70-01, Medellin, Colombia. \\ E-mail: nestor.alvarez@upb.edu.co
}

\section{KEYWORDS \\ RAMS, CAMx, Model, Web-Based Information System.}

\begin{abstract}
This document introduces a Web Based Forecasting Information System. The system gives the user meteorological and chemical (air quality) forecasting information of a defined domain in a web based manner. The user can access through a web page products like: chemical and meteorological forecasting animations, images and text plain data of a given point inside the global domain. All the forecasting data is obtained via the CAMx and RAMS numerical models. The system is actually being used for a government control entity and is actually available at http://simeca.metropol.gov.co.
\end{abstract}

\section{INTRODUCTION}

The main purpose of the prediction system is to inform the users, in real time, about the air quality and meteorological conditions in a defined domain. The system can give air quality and meteorology related information of any point inside the domain, in a graphical and plain text format.

The whole prediction system is indeed a Web Application that is based on tools developed for the visualization and the handling of information product of Meteorological and Chemical Forecasting. The nucleus of the whole system is conformed by a Meteorological Forecasting Model, RAMS (www.atmet.com), and by a Chemical Forecasting Model, CAMx (www.camx.com). A diagram of the entire application can be seen on Figure 1.

When the models have finished making their forecasts, these are generally stored in Data Base which is the central part of the system (see Figure 1), because from this point flows of information towards the graphical tools, and towards the end user of the system. The storage technique for the forecast data depends on the data base capacity, as the growth is exponential.
The system visualizes its results by means of specific visualization tools, designed specifically for the type of information thrown by the system - weather \& air quality forecast information. These tools are:

- $\quad$ Vis5d

(http://www.ssec.wisc.edu/ billh/vis5d.html),

- NCARG

(http://ngwww.ucar.edu/ng4.4/index.html),

- PyNGL

(http://www.pyngl.ucar.edu/).

All these tools work under the UNIX like Operating system, as well as the Forecast Models. The interface between all these tools and the web page was constructed using PHP (Hypertext Preprocessor, www.php.net) programming language.

Finally, the system end user accedes to all the information through a Web Browser. By means of this, the user can obtain graphical, binary and plain text forecast data related to a point inside the global domain. Detailed an up-to-date information about the system can be obtained at http://simeca.metropol.gov.co.

\section{SYSTEM ARCHITECTURE}

The system is basically conformed by three modules: 1) Forecast Cluster, 2) the Web server, and 3) a Data Base.

The communication between the Forecast Cluster and the Web server is established using the SSH (Secure Shell) protocol. SSH is a set of network standards and protocols that allow to establish a safe communication channel between two computers. SSH typically is used to accede to a remote computer and to execute commands, also allows file transfer by means of the associated protocols SFTP (Secure File Transfer Protocol) or SCP (Secure Copy). Using SSH, the Data Base Server obtains the final forecast files from the Forecast Cluster, the Web Server processes them in order to turn them into graphs, plain data and animations (dynamically, depending on user request); and then gives the data to the end user as he requested. 


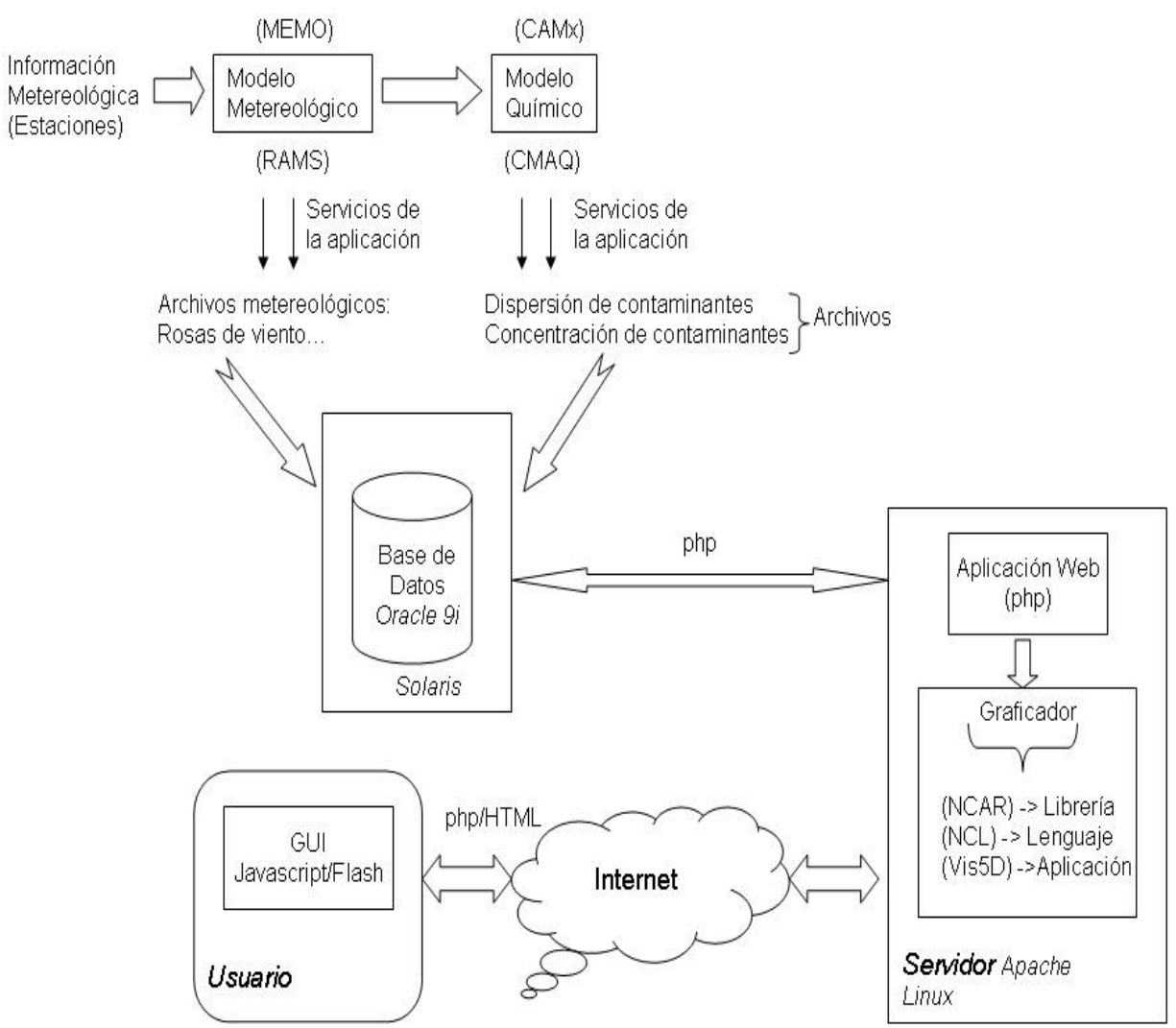

Figure 1. General System Diagram.

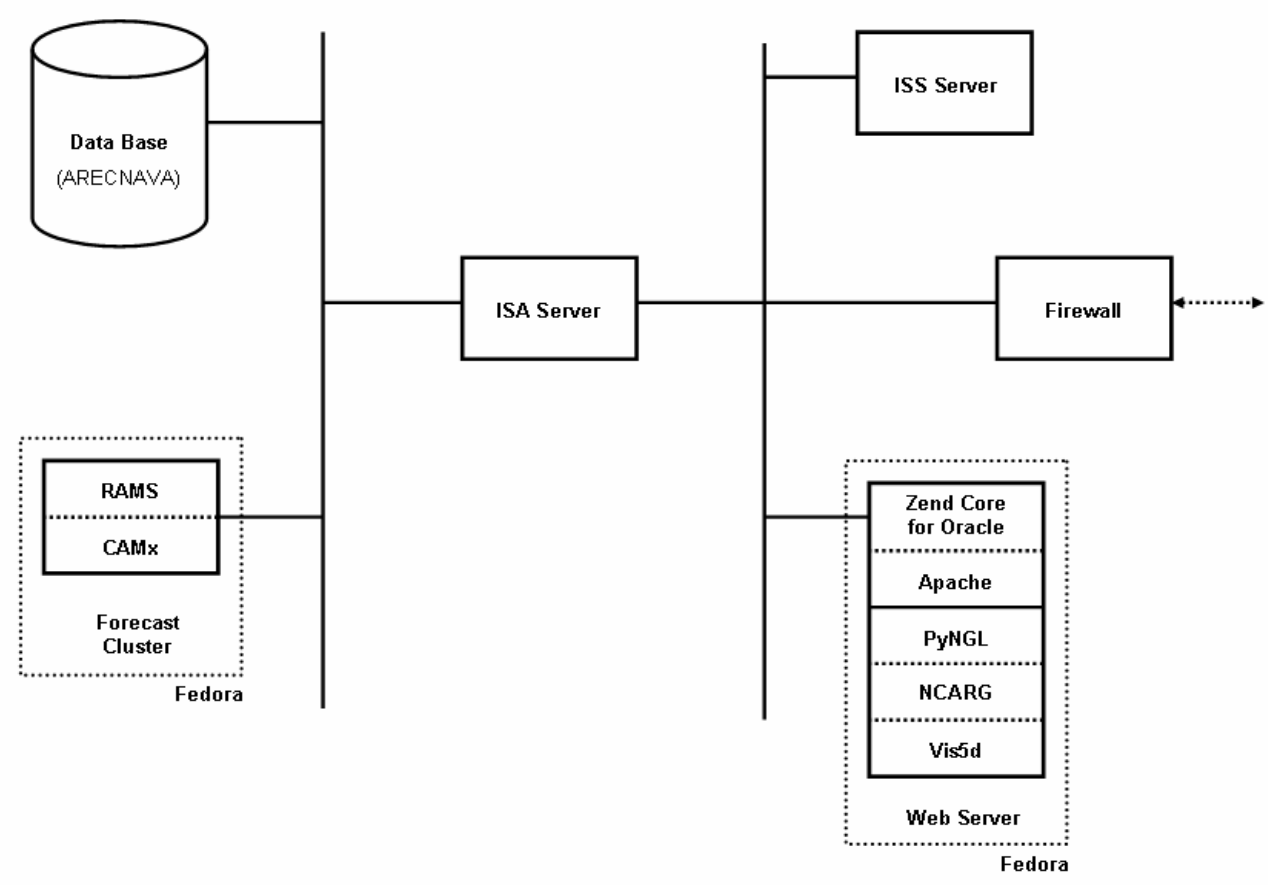

Figure 2. General System Architecture. 
The Figure 2 shows the general system architecture. It's a typical Web Application Architecture. In some blocks, the applications used are shown.

The Communication between the Web server and the Data Base Server is established by means of a clientserver session, where the Web Server acts as client via the "Zend for Core Oracle" Application. Zend Core ${ }^{\mathrm{TM}}$ for Oracle ${ }^{\circledR}$ allows the use of PHP (Preprocessor Hypertext, http://www.php.net/) with the Oracle DBMS (Data Base Management System) in Web Applications; In fact, Zend Core ${ }^{\mathrm{TM}}$ for Oracle ${ }^{\circledR}$ is a PHP Development Environment that allows a complete integration with Oracle data bases.

The system Web services are implemented by means of the conjunction use of the Apache Web Server application (http://www.apache.org/) and PHP as an Application Server. Both, PHP and Apache, run under the Fedora UNIX distribution (http://fedora.redhat.com/). The system access the graphical tools (PyNGL, NCARG, Vis5d) services using PHP, they allow the system to generate the graphics it needs dynamically and in Real Time.

\section{SYSTEM CHARACTERISTICS}

As it was insinuated before, the main system is made up of three subsystems: 1) Forecast Models Module, 2) Data Base Module and 3) Web Services Module.

The Forecast Module was implemented in a Cluster. The Forecast Models (RAMS \& CAMx) are installed on this machine which runs the Fedora Core 4 Operating System. The Forecast Module generates binary archives containing forecast information about the geographical domain selected. These archives are processed by the others modules so that all the services of the system are provided to the end user. All the information output by this module is stored on backup disks. The Module's Cluster is operated via a Front End Machine, usually the slowest machine of the set or any other low resources machine, who allows an operator to control the entire cluster and run the numerical models.

The binary archives obtained from de Forecast Module are used by the Web Services Module to generate graphs, animation and plain text data dynamically. All this is done via the models post-processing tools and state of the art scientific graphical tools. Also, the plain text data can be stored in a Data Base using PHP Programming Language. The binary files are also stored in a repository way for future analysis, they can also be stored in a Data Base or in Backup Disks either magnetic (tapes) or optical disks.

As mentioned early, The Web Services Module was implemented using the Apache Web Server Application in conjunction with a PHP Application Server. Trough PHP scripts and several different tools, the Web
Services Module obtain the necessary data from the Forecast Module's output binary files to generate the data or graphical information requested by the system's end user from a given Web Page. After the user request, the information is displayed to the end user in real time, and all the data requested is generated dynamically.

\section{Modules Information Flow}

The scheme shown in Figure 1 allows to observe how would be the information flow in the entire system. On first place, all the Meteorological Forecasts are done using Reanalysis Data obtained from different Global Models Runs. Then, the data generated for the Meteorological Forecast Model is saved on a data base and used as input to the Chemical Model. All the data is stored in a repository from where the Web Services Module can transform it into graphical and plain text data as end user request. The end user defines the required data characteristic trough a Web Form that is served by a PHP script who transforms the binary data into the format specified by the web page user. The PHP scripts trust on tools as PyNGL, vis5d and REVU (RAMS postprocessor tool) to give the end user the requested data.

\section{System Services Request Procedure}

The end user can access all the services supported by the system trough a typical web page (see Figure 3 ). On this web page the user will find a brief system explanation, system usage tutorials and other useful documentation, and web links. Also, from the system home page the end user can access several web forms from where to interact with the system, one of this web forms is shown on Figure 4. Then in the web form, the end user can define the geographical point of interest (inside the model forecast domain) by clicking one point over a map who represents the models forecast domain or by entering the interest point coordinates (latitude, longitude) in decimal degrees using the web form.

Once the end user have interacted with one of the several web forms, he will be redirected to a web page where all the requested forecast products will be shown, all the products are related to the point first defined by the end user. For a given geographical point, the end user can request products like: XY Plots of a predefined forecast variable, Meteograms, Soundings, among other system products. Also, the end user can choose the final format of the system products requested, it can be graphs, XY plots, 3D animations or plaint text data. At the end of the process, the end user will obtain the required data, in the requested format.

\section{BRIEF TOOLS DESCRIPTION BY MODULE}

Above in this article where described the several modules that conforms the system, now it will be described the various tools that are grouped into each module. 


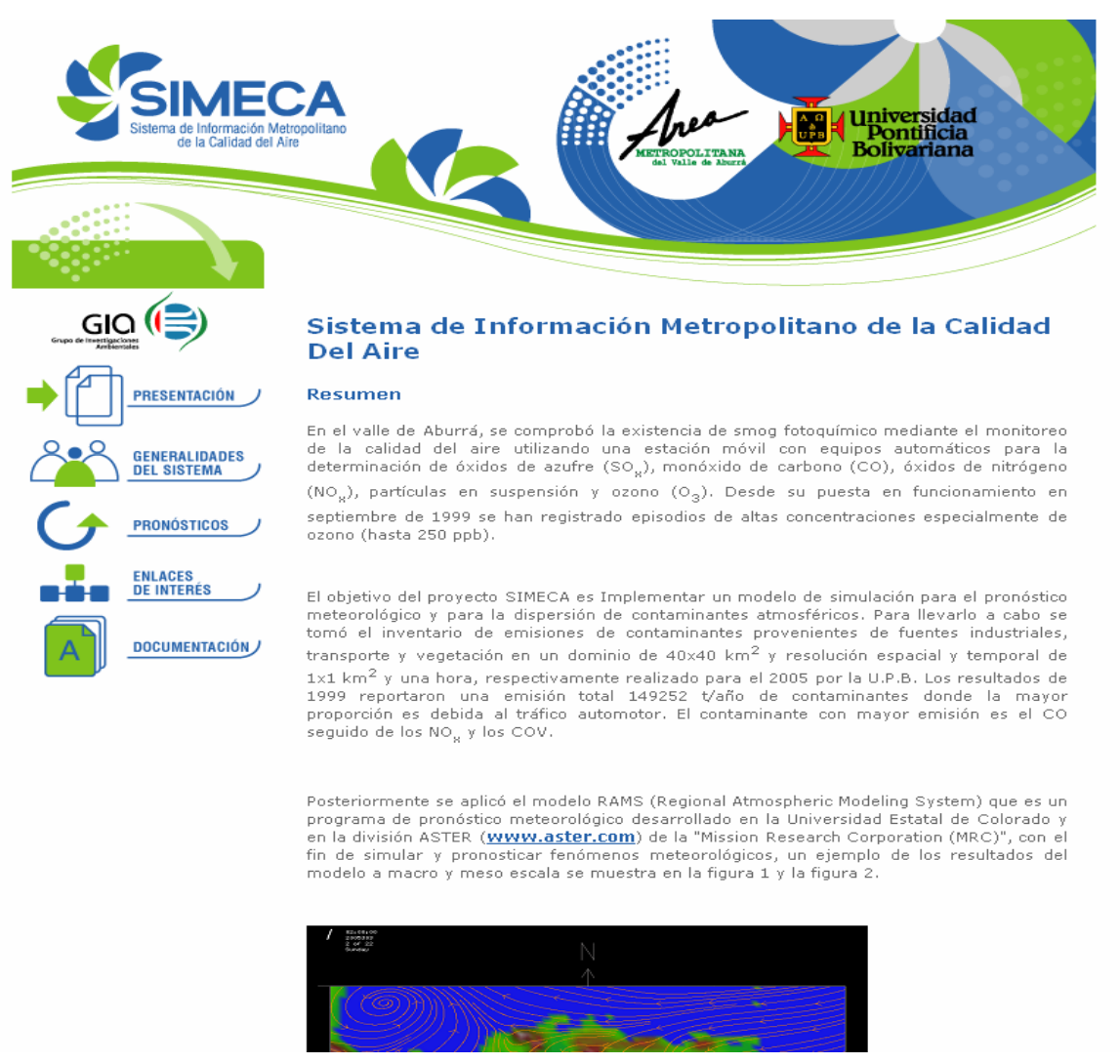

Figure 3. System Home Web Page.

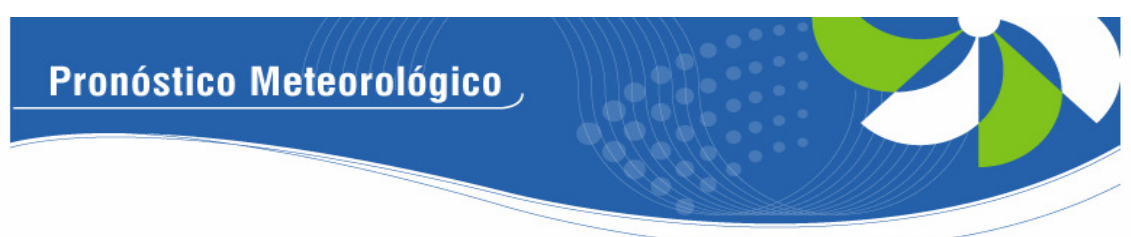

$\underline{\text { Inicio }}{ }^{*}$ Contactos
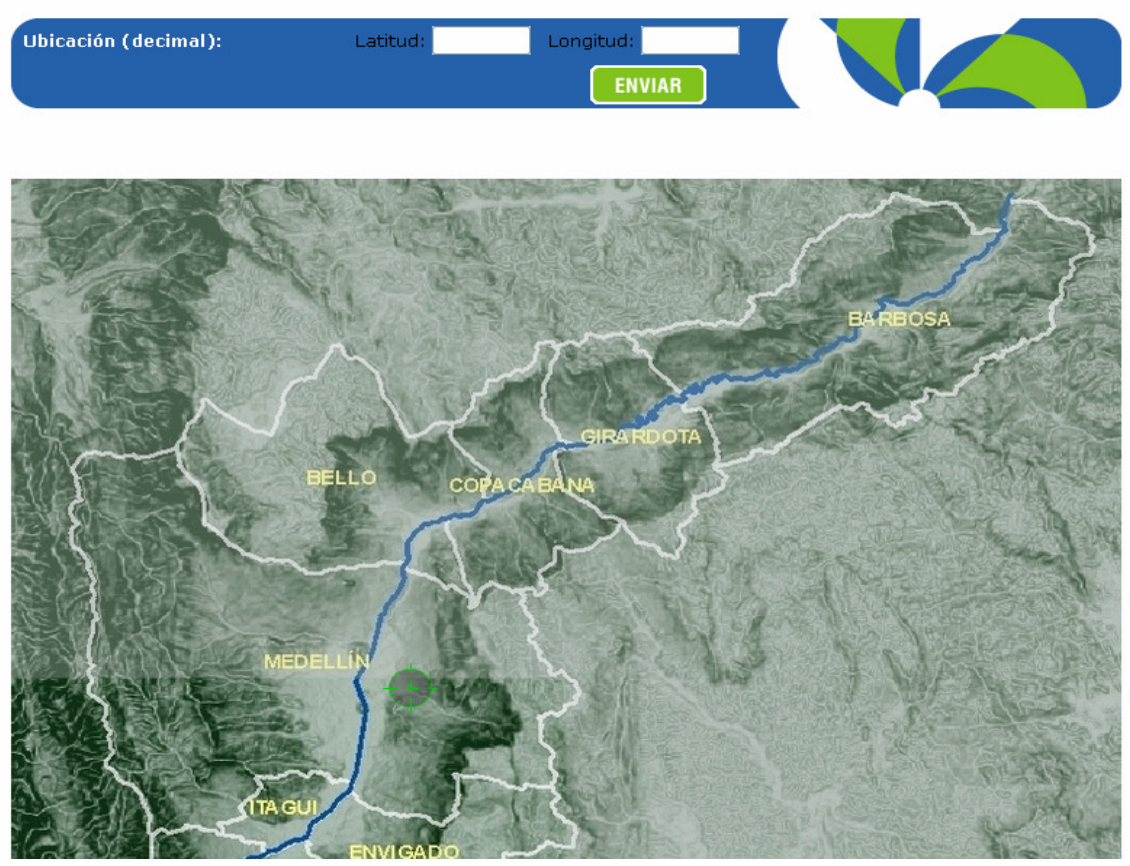

Figure 4. Meteorological Forecast Web Form with an interactive map. 


\section{Forecast Models Module}

This Module is conformed by 2 numerical forecast models and their post-processing \& pre-processing tools. Several of the post \& pre-processing tools where developed by us in order to accomplish all the functional system requirements.

\section{Regional Atmospheric Modeling System (RAMS)}

The Regional Atmospheric Modeling System, is a numerical model developed by scientists at Colorado State University and the ASTER division of Mission Research Corporation for simulating and forecasting meteorological phenomena, and for depicting the results. Its major components are:

(1) an atmospheric model which performs the actual simulations,

(2) a data analysis package which prepares initial data for the atmospheric model from observed meteorological data, and

(3) a post-processing model visualization and analysis package which interfaces the atmospheric model output with a variety of visualization software and other utilities.

RAMS is most often used as a limited area model, and many of its parameterizations have been designed for mesoscale or high resolution cloud scale grids. However, RAMS may also operate as a global scale model for simulating large-scale systems by configuring two hemispheric grids, which use a polar stereographic projection and continually exchange boundary data between them. There is no lower limit to the domain size or to the mesh cell size of the model's finite difference grid; microscale phenomena such as tornadoes and boundary layer eddies, as well as submicroscale turbulent flow over buildings and in a wind tunnel, have been simulated with this code. For more information see www.atmet.com.

\section{Comprehensive Air quality Model with extensions (CAMx)}

The Comprehensive Air quality Model with extensions is a publicly available open-source computer modeling system for the integrated assessment of gaseous and particulate air pollution. Built on today's understanding that air quality issues are complex, interrelated, and reach beyond the urban scale, CAMx is designed to:

- Simulate air quality over many geographic scales

- Treat a wide variety of inert and chemically active pollutants: Ozone, Inorganic and organic PM2.5/PM10; Mercury and toxics.

- Provide source-receptor, sensitivity, and process analyses.

- Be computationally efficient and easy to use
The U.S. EPA has approved the use of CAMx for numerous ozone and PM State Implementation Plans throughout the U.S, and has used this model to evaluate regional mitigation strategies. For more information see www.camx.com.

\section{Data Base Module}

The Forecast Models Module generates great volumes of information that must be stored. All this information is stored in two ways: In a relational Data Base or in a binary files repository.

To implement the data base we used the Oracle DBMS (Data Base Management System), more precisely Oracle Database 10g. Other DBMS can be used to implement this module; we use this particular DBMS due to platform requirements. To fill the data base, we create a PHP script that generates ASCII files from the binary files resulting from both numerical models runs. Then, these ASCII files are introduced to the data base using SQL statements invoked by a PHP script. After this, the Web Services Module can obtain the data it needs via the data base or the binary files repository. For more information about Oracle DBMS see www.oracle.com.

\section{Web Services Module}

Through this module the system can publish all its products and interact with the end user. This module also interacts with the Data Base Module to obtain the information the end user may need.

The system was implemented using the Apache Web Server Application (www.apache.org) and a special tool for Web Applications named Zend Core ${ }^{\mathrm{TM}}$ for Oracle ${ }^{\circledR}$ (www.zend.com). Zend Core ${ }^{\mathrm{TM}}$ for Oracle ${ }^{\circledR}$ supports businesses using PHP with Oracle Database for business-critical Web applications. It provides a stable, high performance, easy-to-install and supported PHP development and production environment fully integrated with the Oracle Database. Zend Core ${ }^{\mathrm{TM}}$ for Oracle ${ }^{\circledR}$ was designed to support Oracle Database 10g, and Apache 2.0.x which is ideal for our Information System requirements. 


\section{REFERENCES}

Sellers, P.J., 1992. "Canopy reflectance, photosynthesis and transpiration. Part III: Areanalysis using enzyme kineticselectron transport models of leaf physiology.Remote Sens." Environ., 42, 187-216.

Sellers, P.J., D.A. Randall, G.J. Collatz, J.A. Berry, C.B. Field, D.A. Dazlich, C. Zhang, G.D. Collelo, and L. Bounoua, 1996. "A revised land surface parameterization (SiB2) for atmospheric GCMs. Part I: Model formulation." J. Climate, 9, 676-705.

Walko, R.L., L.E. Band, J. Baron, T.G.F. Kittel, R. Lammers, T.J. Lee, D. Ojima, R.A. Pielke, C. Taylor, C. Tague, C.J. Tremback, and P.L. Vidale, 2000. "Coupled atmospherebiophysics-hydrology models for environmental modeling.” J. Appl. Meteor., 39, 931-944.

Jacobs, C.A. J.P. Pandolfo and M.A. Atwater, 1974. "A description of a three dimensional numerical simulation model of a coupled air-water and/or air-land boundary layer." IFGYL final report. CEM report No. 5131-509a.

Mesinger, F. and A. Arakawa, 1976. "Numerical Metods used in atmospheric Models." GARP Publication series, No. 14, WMO/ICSU Joint Organizing Committee. 64 pp.

Tremback C. J., Walko R. L. and Bell M. J. 2001 RAMS/HYPACT Evaluation and Visualization Utilities (REVU) Version 2.3.1. User's Guide, *ASTER Division Mission Research Corporation. P.O. Box 466, Fort Collins, CO 80525-0466.

Walko R. L. and Tremback C. J. Regional Atmospheric Modeling System MODEL INPUT NAMELIST PARAMETERS Version 4.3/4.4. [Online] www.atmet.com.

ENVIRON International Corporation. 2006. Comprehensive Air Quality Model with extensions User's guide. ENVIRON International Corporation, 101 Rowland Way, Suite 220. Novato, California 94945-5010.

\section{AUTHOR BIOGRAPHIES}

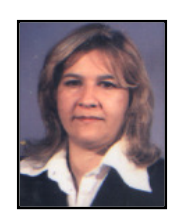

MARIA V. TORO was born in Medellin, Colombia and went to the Bolivariana Pontifical University of Medellin, where she studied Chemical Engineering and obtained his degree in 1988. Then, she worked for the Bolivariana Pontifical University R\&D Institute, specifically at the Environmental Research Group (GIA) as Researcher. In 1991, he went to the Polytechnical University of Madrid, where she obtains a MsC. degree in Environmental Contamination. Later, she returns to the Pontifical University of Medellin where she worked as the director of the Environmental Research Group (GIA) until 1999. The she returns to Spain and obtains a $\mathrm{PhD}$ degree in the Polytechnical University of Catalonia in 2004. Since then, she works for the Pontifical University of Medellin as director of the Atmospherical Research Study Line of the Environmental Research Group (GIA). His e-mail address is: victoria.torodupb.edu.co.

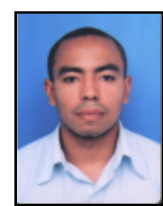

NESTOR W. ALVAREZ was born in Medellin, Colombia and went to the Bolivariana Pontifical University of Medellin, where he studied Electronic
Engineering and obtained his degree in 2002. Since then, he works for the Bolivariana Pontifical University R\&D Institute, specifically at the Environmental Research Group (GIA) in his Atmospherical Research Study Line. Here he dedicates to implement platforms for various types of numerical weather forecast models (RAMS, MEMO, MARS, CAMx, and MM5). His email address is: nestor.alvarezaupb.edu.co and his Web-page can be found at http://www. waldyd. $8 \mathrm{~m} \cdot \mathrm{com} /$.

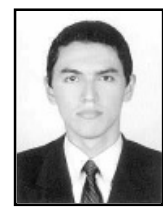

CARLOS G. SANCHEZ TORO was born in Medellin, Colombia, and went to the Bolivariana Pontifical University of Medellin, where he studied Electronic Engineering and obtained his degree in 2004. In 2005, he began to work at the Environmental Research Group (GIA) of the same university, focus himself in software development and web programming through several research projects. His e-mail address is: cg2100 ahotmail.com. 